Polymer science

\section{Soluble conductors on the way}

\author{
Paul Calvert
}

Conducting polymers came to prominence in the mid-1970s, with many promising applications apparent, the most obvious being their use as cheap and light plastic conductors, organic replacements for copper. It soon became obvious that the materials available could not fulfill this role and interest in their use in solar cells, semiconductor devices and batteries grew instead. These now appear to be equally elusive. Four years ago I wrote a News and Views article ${ }^{1}$ explaining why polymers could not be both soluble and conducting and, as a result, that the optimum way to form these materials was from a precursor polymer that could be readily cast into films or spun into fibres. Recent papers describing soluble polythiophenes ${ }^{2,3}$ prove me wrong.

The prototypical conducting polymer is polyacetylene ( $a$ in the figure), where the conjugated double bonds along the chain allow delocalization and mobility of electrons, but also lock the chain into a rigid zig-zag. Such a rigid structure gains little entropy on dissolution or melting, and so remains stubbornly solid until it decomposes. Polyacetylene itself is semiconducting but oxidation or reduction (doping) can remove or add electrons in the delocalized $\pi$-orbitals with the result that the polymer conducts like a metal. Polyphenylene ( $b$ in the figure) followed polyacetylene as the popular polymer to study and is also extremely insoluble, though the reasons are less clear, as the undoped state probably has little $\pi$ orbital overlap between the rings. Polyphenylene differs significantly from polyacetylene in this respect, as shifting all the double bonds sideways one 'step' in polyacetylene produces no change in structure or energy, whereas the same shift in polyphenylene, so that the rings are connected by double bonds, produces a quinoid structure which has a higher energy.

Polypyrrole ( $c$ in the figure) and polythiophene $(d)$ also became the subject of intense interest and were equally insoluble. Both these polymers are related to a trans-cisoid form of polyacetylene. Thus it seemed clear that conduction requires a conjugated structure, but this in turn makes the chain rigid and insoluble.

\section{Corrigendum}

IN the article by Noburu Miura on hightemperature superconducting ceramics (Nature 326, 638-639; 1987) Dr Jaw-Shen Tsai of NEC Corporation was incorrectly called $\mathrm{Dr}$ Aoi. Dr Hikami of Tokyo University was also incorrectly spelt as Dr Hikama.

To the polymer scientist, this is a nuisance as the polymers cannot be purified or properly characterized.

There have been a few reports of soluble states. One method is to 'carry' the polyacetylene into solution by forming a block or graft copolymer with a soluble polymer such as polystyrene. The result, however, is essentially a micellar solution, the polystrene surrounding the polyacetylene, and hence a low-conductivity state of polyacetylene ${ }^{4-6}$. Polyphenylenesulphide and polyacetylene form solutions when doped with arsenic pentafluoride in$$
a
$$<smiles>C=C/C=C/C=C/C=C/C=C/C=C/C</smiles>

arsenic trifluoride, but this is hardly a convenient solvent system for study and irreversible reactions occur with the dopant ${ }^{7.8}$.

Work on polythiophene led to 3-methyl and 3-methoxythiophenes, which are also insoluble ${ }^{9,10}$. The polythiophenes do have the advantage that they are relatively stable compared with polyacetylene and retain their conductivity over several months in air. A group at the Research Institute for Polymers and Textiles in Tsukuba now report ${ }^{2}$ that 3-hexyl and 3eicosylthiophenes make soluble polymers that can be dissolved in the neutral or doped (and conducting) states. They can be doped to give conductivities of 5-100 $\mathrm{S} \mathrm{cm}{ }^{-1}$, comparable to graphite. These polymers are formed by electrochemical polymerization. A group at Allied Chemical obtained similar results ${ }^{3}$ with studies of chemically polymerized thiophenes including 3-butyl and butyl-methyl copolymers. Poly-3-butylthiophene has a glass transition at $48{ }^{\circ} \mathrm{C}$, making it a fairly rubbery polymer rather than a brittle, intractable structure as we have come to expect. The solubility allows molecular weights to be measured: these are rather low for real polymers but still quite respectable, with polymerization of $200-300$ thiophene units. Clearly this is only the start; there are a mass of substituted thiophene and pyrrole polymers, copolymers and composites to work through before the limits of this class of materials are known.<smiles></smiles>

Structures of four conducting polymers. $a$, Polyacetylene; $b$, polyphenylene; $c$, polypyrrole; $d$, polythiophene: $a$ shows the zig-zag arrangement of carbon atoms found in trans structures; $c$ and $d$ show the crenulated arrangement found in trans-cisoid structures, seen by covering the NH or S.<smiles>C[C@H]1CC[C@@H]([C@H]2CC[C@@H](C3CC[C@@H](C)N3)N2)N1</smiles>

At the recent American Chemical Society meeting in Denver (5-10 April), Alan Heeger of the University of California, Santa Barbara, described the spinning of fibres of soluble polythiophenes in blends with polystyrene or polyethylene oxide, and Herbert Naarman of BASF in Ludwigshafen described methods for drawing doped polyacetylene to give conductivities above $10^{5} \mathrm{~S} \mathrm{~cm}^{-1}, 100$ times higher than usually found and comparable to copper. Heeger, Naarman and collaborators report their results on page 403 of this issue ${ }^{\mathrm{t1}}$.

The surprising conclusion is that conductivities measured so far have been limited by the microstructure or perfection of the samples and the real limits may be higher still. The same could be applied to the low conductivities found for polythiophenes. M. Bryce and co-workers at

Durham University recently reported ${ }^{12}$ an ether-substituted compound with conductivity greater than $1,000 \mathrm{~S} \mathrm{~cm}^{-1}$.

It is easier to develop theories when the experimental answers are already known, and it should not be too difficult now to reconcile the twin requirements for conjugation and flexibility in these polymers. Since conducting polymers first excited interest, there have been many disappointments as various leads seemed to fade away. Now we are back with the original concept, a (more or less) stable, processible, film-forming, organic conductor. There is always a catch, but for now the field has a new dawn.

1. Calvert, P. Nature 304, 487 (1983).

2. Sato, M., Tanaka, S. \& Kaeriyama, K. JCS Chem. Commun. 873-874 (1986)

3. Jen. K.-Y., Miller, G.G. \& Elsenbaumer, R.L. JCS Chem. Commun. 1347-1348 (1986).

4. Bates, F.S. \& Baker, G.L. Macromolecules 16. 704-707 (1983)

5. Bates, F.S. \& Baker, G.L. Macromolecules 16, 1013-1015 (1983)

6. Bates, F.S. \& Baker, G.L. Macromolecules 17, 2619-2626 (1984)

7. Frommer, J.E., Elsenbaumer, R.L. \& Chance, R.R. Am Chem. Soc. Symp. 242, 447-453(1985)

8. Aldissi, M. Polym. Prepr. 26 No. 2, 269-270 (1985).

9. Tourillon, G. \& Garnier, F. J. polym. Sci. (Phys.) 22, 33-39 (1984).

10. Tanaka, S., Sato, M. \& Kaeriyama, K. Polym. Commun 26, 303-306 (1985).

11. Basescu N. et al. Nature 327, $403-405$ (1987)

2. Bryce, M.R., Chissell A., Kathirgamanathan, P., Parker D.\& Smith, N.R.M. JCS Chem. Commun. 466-467 (1987).

Paul Calvert is in the School of Chemistry and Molecular Sciences, University of Sussex, Falmer, Brighton BN1 9QJ, UK. 